\title{
Aspectos endoscópicos da habronemose gástrica equiina*
}

\section{Endoscopic aspects of equine gastric habronemosis}

\section{Aspectos endoscópicos de la habronemosis gástrica equina}

\author{
Carla Bargi Belli; ${ }^{1}$ Luís Cláudio Lopes Correia da Silva; ${ }^{2}$ Wilson Roberto Fernandes ${ }^{3}$
}

Departamento de Clínica Médica da Faculdade de Medicina Veterinária e Zootecnia da Universidade de São Paulo (FMVZ/USP). São Paulo, SP, Brasil

\section{Resumo}

Objetivo: O objetivo deste estudo foi verificar a ocorrência de Habronema sp. em eqüinos sadios e comprovar a possibilidade de utilização da gastroscopia como seu método de diagnóstico. Material e Método: Foi realizado exame gastroscópico em 21 eqüinos sadios, de diversas raças, com idade entre 3 e 20 anos, provenientes do Serviço de Clínica Médica de Eqüinos da Faculdade de Medicina Veterinária e Zootecnia da Universidade de São Paulo, São Paulo, SP. Resultados: A ocorrência de Habronema sp. foi de $28,6 \%$, principalmente junto ao margo plicatus. A presença de quantidade excessiva de muco foi identificada em $50 \%$ dos animais parasitados. Os parasitas recuperados foram identificados como Habronema muscae. Conclusões: O Habronema sp. apresenta alta ocorrência no Brasil. Endoscopicamente, a habronemose gástrica é caracterizada pela presença de gastrite catarral, com excesso de produção de muco, e presença dos parasitas. Embora infecções com baixa carga parasitária possam não ser identificadas, a gastroscopia revelou-se uma forma eficiente de diagnóstico de habronemose gástrica em eqüinos.

Palavras-chave: Infecções por Spirurida, diagnóstico. Spiruroidea, patogenicidade. Endoscopia do sistema digestório. Gastroscopia. Gastrite. Estômago. Equidae.

\footnotetext{
' Pós-graduanda do Departamento de Clínica Médica da FMVZ/USP. CRMV-SP 10096

${ }^{2}$ Professor Doutor do Departamento de Cirurgia da FMVZ/USP. CRMV-SP 05993

Professor Doutor do Departamento de Clínica Médica da FMVZ/USP. CRMV-SP 03034

* Auxílio financeiro da Fundação de Amparo à Pesquisa do Estado de São Paulo (FAPESP). Processo 99/00225-3

Parte de Dissertação de Mestrado apresentada à Faculdade de Medicina Veterinária e Zootecnia da Universidade de São Paulo, São Paulo, 2001

Publicado em: CONGRESSO BRASILEIRO DE MEDICINA VETERINÁRIA, 28., 2001, Salvador. Anais... p. 125
} 


\section{Introdução}

O Habronema sp. é um nematóide de distribuição mundial que parasita a porção glandular do estômago dos eqüídeos, principalmente próximo ao margo plicatus, ${ }^{1-3}$ possuindo de 1 a $2,5 \mathrm{~cm}$ de comprimento. ${ }^{3,4}$

As principais espécies são o $H$. muscae e o $H$. microstoma. ${ }^{2-4}$ E improvável serem confundidos com outros nematóides do estômago, pois a Draschia megastoma está associada à formação de grandes nódulos fibrosos e o Trichostrongylus axei tem menos de $1 \mathrm{~cm}$ de comprimento. ${ }^{3}$

Os hospedeiros intermediários deste parasita são a Musca domestica (principalmente para o $H$. muscae) e a Stomoxys calcitrans. ${ }^{1,2,5}$ Os adultos do Habronema sp. vivem livres no estômago, em uma camada de muco que adere na mucosa, e podem ou não invadir as glândulas gástricas. ${ }^{1,5,6} \mathrm{O}$ Habronema muscae também pode invadir o pulmão, causando nódulos que se manifestam como bronquite. ${ }^{1}$ As larvas também podem ser encontradas no pulmão de potros, associadas com abscessos por Rhodococcus equi. ${ }^{6}$ Quando as larvas são depositadas sobre uma ferida cutânea ou ao redor dos olhos, elas invadem os tecidos, mas não completam seu desenvolvimento. ${ }^{3}$

As manifestações clínicas são raras, ${ }^{1-3,7}$ já que parasitas gástricos e corpos estranhos não são causas comuns de ulceração gástrica em eqüinos. ${ }^{8}$ Mas o Habronema sp. pode irritar superficialmente a mucosa gástrica e estimular hipersecreção de muco viscoso e espesso (por hiperplasia das células produtoras de muco), levando a uma gastrite catarral crônica de certa gravidade. . $^{2-5}$ Estudos também comprovaram que a presença desse parasita no estômago age como fator predisponente ao aparecimento das úlceras gástricas em eqüinos quando associadas a outros fatores. ${ }^{9}$ Sua principal importância clínica reside no fato de suas larvas poderem invadir os olhos (causando conjuntivite) e feridas na pele, causando a habronemose cutânea ou "ferida de verão", muito comum no Brasil..$^{2-4,7,10}$

A infecção gástrica dificilmente é diagnosticada, já que os ovos e larvas não são demonstrados facilmente nas fezes por técnicaspadrão de flutuação. 1,3,5,7 Outras formas de diagnóstico com o animal vivo, como o xenodiagnóstico $^{1,2}$ e a detecção de ovos e parasitas por lavagem gástrica, ${ }^{4-6}$ são possíveis, mas com pouca praticidade e eficiência.

O uso da gastroscopia para diagnóstico de parasitismo gástrico em eqüinos é citado na literatura, ${ }^{11}$ embora não exista nenhum trabalho específico com essa finalidade.

Os estudos de prevalência descritos na literatura são todos estudos necroscópicos. Em trabalho na Normandia com 410 eqüinos com mais de dois meses de idade, a prevalência de Habronema sp. foi de $8,5 \%{ }^{12}$ No Brasil, uma avaliação necroscópica de 37 eqüinos, 18 asininos e 22 muares oriundos de oito estados revelou $H$. muscae em metade dos animais e H. microstoma em um terço deles. ${ }^{13}$ Em outro estudo brasileiro, no estado do Rio de Janeiro, com 36 eqüinos e 4 asininos, a prevalência encontrada foi de $90 \%$ para $H$. muscae e de $65 \%$ para H. microstoma. ${ }^{14}$

A alta prevalência encontrada no Brasil evidencia um controle parasitário deficiente, já que a maioria dos anti-helmínticos modernos de amplo espectro têm atividade contra os parasitas adultos no estômago, ${ }^{3}$ sendo o tratamento com ivermectina $(0,2$ $\mathrm{mg} / \mathrm{kg}$, via oral) bastante eficaz. ${ }^{2,7}$

A prevenção da infecção deve ser feita pelo combate às moscas (destino adequado das fezes, uso de inseticidas, repelentes tópicos) e vermifugação. ${ }^{1-3,5}$

O objetivo do presente estudo foi verificar a ocorrência de Habronema sp. em eqüinos sadios, as alterações gástricas causadas por este parasitismo, além de comprovar a possibilidade de utilização da gastroscopia como método de diagnóstico da habronemose gástrica.

\section{Material e Método}

Foi realizado exame gastroscópico em 21 eqüinos sadios, de diversas raças, com idade entre 3 e 20 anos, atendidos no Serviço de Clínica Médica de Eqüinos da 
Faculdade de Medicina Veterinária e Zootecnia da Universidade de São Paulo, São Paulo, SP.

Sem se levar em consideração o histórico de vermifugação, foi realizado exame coproparasitológico de todos os animais pela contagem de ovos por grama de fezes (OPG), com o método modificado de MacMaster.

Após jejum, sedação e contenção física, um videogastroscópio, marca Karl Storz GMBH \& Co., com $3 \mathrm{~m}$ de comprimento útil e $14 \mathrm{~mm}$ de diâmetro foi introduzido até o estômago dos animais. Ao chegar na luz gástrica, esta foi dilatada com ar até se obter a melhor visualização possível sem causar desconforto ao animal.

Observou-se, então, toda a mucosa aglandular, o margo plicatus e a porção visível da mucosa glandular. Por intermédio da inspeção da mucosa glandular e colheita com pinça, procurou-se identificar a presença de Habronema sp., recolher parasitas de alguns animais para identificação e registrar imagens dos estômagos parasitados e lesões relacionadas.

Foi realizada comparação de médias dos valores de OPG entre os animais com e sem a presença de Habronema sp., considerando-se $\mathrm{p} \leq 0,05$ para indicar diferença estatística entre os grupos. Utilizou-se para esta avaliação o programa de computador GrafPad InStat ${ }^{T M}$ (GraphPad Software, versão 3.01, 1998).

\section{Resultados}

Foi possível identificar a presença de Habronema sp. por meio da gastroscopia, e a ocorrência encontrada de 28,6\% (6 animais de 21). A principal observação para indicar a presença do parasita foi a visualização de helmintos brancos e finos movimentando-se sobre a superfície da mucosa glandular, cobertos ou não por muco, podendo elevarse em direção à luz gástrica. Em casos de dúvida, quando não havia movimentação visível, procurouse também retirar os parasitas da mucosa com pinça de biópsia. A área onde os parasitas foram visualizados com maior facilidade e em maior quantidade foi a região junto ao margo plicatus.

Os parasitas recuperados foram identificados como Habronema muscae. A presença de quantidade excessiva de muco foi constatada em 50\% dos animais parasitados. Em alguns animais foi possível identificar grande quantidade de parasitas dentro da camada de muco (Figura 1).

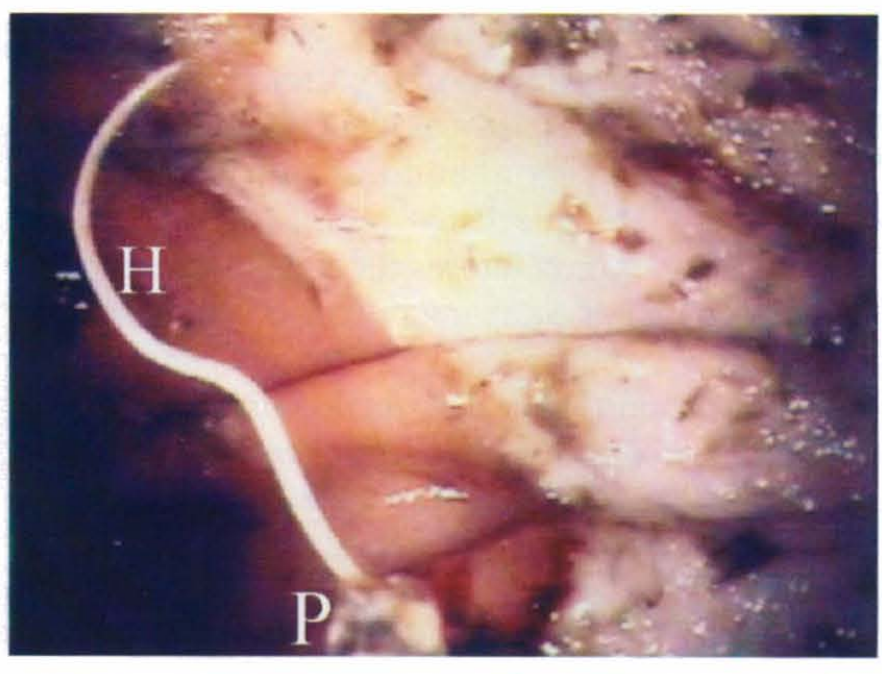

Figura 1 - Imagem endoscópica de Habronema sp. (H) sendo retirado por pinça de biópsia (P)

Dois eqüinos parasitados (33,3\%) apresentaram gastrite (leve e moderada) na mucosa glandular, e nenhum dos animais com aumento de muco apresentou gastrite. A gastrite aqui citada refere-se apenas à visualização endoscópica de avermelhamento da mucosa. Nenhum animal revelou ulcerações na mucosa glandular.

\section{Discussão}

A gastroscopia foi facilmente realizada, não apresentando dificuldades na visualização da região junto ao margo plicatus, onde ocorre maior concentração do Habronema sp. A própria visualização dos parasitas é simples, sendo possível observá-los movimentando-se sob o muco, elevandose em direção à luz gástrica ou por sua retirada com pinça de biópsia. Confirmando as descrições de literatura, ${ }^{3,4}$ não há como confundi-los com outros parasitas gástricos. 
A observação de quantidade de muco elevada ou gastrite da mucosa glandular nos animais com presença gástrica de Habronema sp. está de acordo com a literatura, que cita este parasita como causador de gastrite catarral com excesso de produção de muco. ${ }^{2,3}$ A observação dos parasitas dentro da camada de muco e junto ao margo plicatus também coincide com as descrições de literatura. ${ }^{1-3,5,6}$

Apesar da presença de gastrite catarral, a habronemose gástrica não foi correlacionada com a presença de úlceras gástricas glandulares nos animais avaliados, correspondendo ao que é citado na literatura, visto que estes animais não estavam submetidos a outros fatores ulcerogênicos, fato que poderia predispor ao aparecimento das úlceras. ${ }^{9}$

Uma ocorrência de parasitismo menor que a encontrada por outros autores no Brasil sugere, além de diferenças regionais e de manejo dos animais, uma possível deficiência da gastroscopia em detectar baixas cargas parasitárias. Isso ocorre porque os parasitas, quando em pequeno número, podem passar despercebidos ao se movimentarem na mucosa durante o exame, além de não causarem alterações significativas naquela.

\section{Conclusões}

O Habronema sp. apresenta alta ocorrência no Brasil. Endoscopicamente, a habronemose gástrica é caracterizada pela presença de gastrite catarral, com excesso de produção de muco, e presença dos parasitas. Embora não seja uma técnica de rotina e infecções com baixa carga parasitária possam não ser identificadas, a gastroscopia revelou-se uma técnica fácil e eficiente na detecção da habronemose gástrica e das suas conseqüências sobre a mucosa do estômago dos eqüinos.

\section{Abstract}

Objective: The purpose of this study was to investigate the occurrence of Habronema sp. in healthy horses and to confirm the effectiveness of gastroscopy as a diagnostic method. Material and Method: Gastroscopic examination was performed in 21 healthy horses of different breeds and aged between 3 and 20 years, referred from the Equine Clinic Service of the Faculdade de Medicina Veterinária e Zootecnia da Universidade de São Paulo (School of Veterinary Medicine and Animal Science of the University of São Paulo), São Paulo, SP, Brazil. Results: Habronema sp. was present in $28,6 \%$ of the horses, mostly close to the margo plicatus. The presence of excessive amounts of mucus was documented in 50\% of the affected animals. The parasites recovered were identified as Habronema muscae. Conclusions: Habronema sp. is common in Brazil. Endoscopically, gastric habronemosis is characterized by the presence of the parasites and an associated catarrhal gastritis, with excessive mucus production. Although infections with low parasitic load may go unidentified, gastroscopic examination is an efficient diagnostic method for gastric habronemosis in horses.

Keywords: Spirurida infections, diagnosis. Spiruroidea, pathogenicity. Endoscopy, digestive system. Gastroscopy. Gastritis. Stomach. Equidae. 


\section{Resumen}

Objetivo: Verificar la presencia de Habronema sp. en equinos sanos y confirmar la posibilidad de la utilización de la gastroscopia como método de diagnóstico. Material y Método: Fueron realizados exámenes gastroscópicos en 21 equinos sanos de varias razas, con edad entre 3 y 20 años, y que provenían del "Serviço de Clínica Médica de Eqüinos" de la Facultad de Medicina Veterinaria y Zootecnia de la Universidad de São Paulo, SP, Brasil. Resultados: La presencia de Habronema sp. fue de un 28,6\%, principalmente junto al margo plicatus. La presencia de cantidad excesiva de moco se ha identificado en un $50 \%$ de los animales infectados. Los parásitos rescatados fueron identificados como Habronema muscae. Conclusiones: El Habronema sp. es bastante frecuente en nuestro país. Endoscópicamente, la habronemosis gástrica se caracteriza por la presencia de gastritis catarral, con exceso de producción de moco, y presencia de los parásitos. Aunque infecciones con baja carga parasitaria puedan no ser identificadas, la gastroscopia mostró ser una forma eficiente de diagnóstico de habronemosis gástrica en equinos.

Palabras-clave: Infecciones por Spirurida, diagnóstico. Spiruroidea, patogenicidad. Endoscopia del sistema digestivo. Gastroscopia. Gastritis. Estómago. Equidae.

\section{Referências}

1. FORTES, E. Subfamília Habronematinae. In: FORTES, E. (Ed). Parasitologia veterinária. São Paulo: Ícone, 1997. p. 384-391.

2. THOMASSIAN, A. Parasitos do estômago e intestinos. In: Enfermidades dos cavalos. 2. ed. São Paulo: Varela, 1997. p. 357-367.

3. URQUHARD, G. M. et al. Helmintologia veterinária. In: Parasitologia veterinária. Rio de Janeiro: Guanabara Koogan, 1990. p. 3-145.

4. LEITÃO, J. S. Espirurídeos. In: Parasitologia veterinária. 3. ed. Lisboa: Fundação Calaouste Gulbenkian, 1983. p. 156-158.

5. BLAGBURN, B. L. et al. Pathogenesis, treatment and control of gastric parasites in horses. Compendium on Continuing Education for the Practicing Veterinarian, v. 13 , p. $850-857$, 1991.

6. AIELLO, S. E.; MAYS, A. Parasitas gastrointestinais dos eqüinos. In: AIELLO, S. E.; MAYS, A. (Ed.). Manual Merck de veterinária. 8. ed. São Paulo: Roca, 2001. p. 165-170.
7. ROSE, R. J.; HODGSON, D. R. Sistema alimentario. In: Manual clinico de equinos. México: Interamericana-McGraw-Hill, 1995. p. 232-301.

8. DEARO, A. C. O.; LOPES, M. A. F. Úlcera gastroduodenal em potros. Ciência Rural, v. 25, p. 323-330, 1995.

9. SILVA, L. C. L. C.; ALVARENGA, J.; KLEMM, M. Efeitos de drogas antiinflamatórias não esteroidais e estresse sobre a mucosa gástrica de eqüinos. Brazilian Journal of Veterinary Science, v. 9, p. 92-94, 2002.

10. SLOCOMBE, J. O. D. Pathogenesis of helminths in equines. Veterinary Parasitology, v. 18, p. 139-153, 1985.

11.BROWN, C. M.; SLOCOMBE, R. F.; DERKSEN, F. J. Fiberoptic gastroduodenoscopy in the horse. Journal of American Veterinary Medical Association, v. 186, p. 965-968, 1985.

12.COLLOBERT-LAUGIER, C. et al. Prevalence of stomach nematodes (Habronema sp., Draschia megastoma and Trichostrongylus axei) in horses examined post mortem in Normandy. Revue de Médecine Vétérinaire, v. 151, p. 151-156, 2000. 
13.SILVA, A. V. M.; MORAES, C. F. A.; COSTA, H. M. A. Habronema e Draschia: alguns dados sobre infecções em eqüinos, asininos e muares de alguns estados brasileiros. Brazilian Journal of Veterinary Research and Animal Science, v. 33, p. 89-96, 1996.
14.LEITE, J. O. et al. Prevalência de helmintos gástricos de eqüídeos da região metropolitana do Rio de Janeiro. Revista Brasileira de Parasitologia Veterinária, v. 6, p. 75-78, 1997. 